\title{
Valence modulates source memory for faces
}

\author{
RaOul Bell ANd AXel Buchner \\ Heinrich-Heine-Universität Düsseldorf, Düsseldorf, Germany
}

\begin{abstract}
Previous studies in which the effects of emotional valence on old-new discrimination and source memory have been examined have yielded highly inconsistent results. Here, we present two experiments showing that old-new face discrimination was not affected by whether a face was associated with disgusting, pleasant, or neutral behavior. In contrast, source memory for faces associated with disgusting behavior (i.e., memory for the disgusting context in which the face was encountered) was consistently better than source memory for other types of faces. This data pattern replicates the findings of studies in which descriptions of cheating, neutral, and trustworthy behavior were used, which findings were previously ascribed to a highly specific cheater detection module. The present results suggest that the enhanced source memory for faces of cheaters is due to a more general source memory advantage for faces associated with negative or threatening contexts that may be instrumental in avoiding the negative consequences of encounters with persons associated with negative or threatening behaviors.
\end{abstract}

The ability to remember is the product of evolution. To remember the past is adaptive, because it can help us to gain control over the present and the future (Nairne \& Pandeirada, 2008a, 2008b; Nairne, Pandeirada, \& Thompson, 2008; Nairne, Thompson, \& Pandeirada, 2007). However, how the cognitive architecture is designed to achieve specific adaptive ends is subject to debate. A popular assumption in evolutionary psychology is that the human mind is composed of highly specialized modules that have evolved to solve the problems of survival and reproduction faced by our ancestors during the course of evolution. A prominent example is the cheater detection module postulated by social contract theory (Cosmides, 1989; Cosmides \& Tooby, 1992, 2005). According to this theory, cheater detection is an evolutionary necessity, because social cooperation cannot emerge unless cooperators have evolved a look-for-cheaters mechanism that enables them to reliably detect cheaters and, thus, avoid exploitation. The Wason (1968) selection task was repeatedly used to seek evidence in support of social contract theory (Cosmides, 1989; Cosmides \& Tooby, 1992, 2005). A continuing controversy is whether these findings can be best ascribed to a cheater detection module or to more domain-general processing mechanisms (e.g., Carlisle \& Shafir, 2005; Fiddick \& Rutherford, 2006).

A strategy to avoid being consistently exploited by cheaters consists of refusing to cooperate with individuals who have previously cheated (reciprocal altruism; Axelrod \& Hamilton, 1981; Trivers, 1971). This requires good memory for cheaters, in addition to cheater detection. More specifically, it has been suggested that the ability to recognize faces of cheaters may be important in this respect. Seemingly consistent with these assumptions,
Mealey, Daood, and Krage (1996) reported that for faces associated with low-status professions, old-new discrimination was better for faces associated with descriptions of cheating than for faces associated with trustworthy behavior. Oddly, this result was not found for faces associated with high-status professions. More recent studies have been unable to replicate the Mealey et al. finding (Barclay \& Lalumière, 2006; Mehl \& Buchner, 2008). This is not unexpected from a functional perspective on human memory, however, because just perceiving a face as familiar, without concurrent memory for the context in which a face was encountered, cannot be of help in avoiding cheaters and, thus, cannot provide an evolutionary benefit. Even worse, increased familiarity of faces of cheaters without context information might increase the risk of being exploited, because of the preference often exhibited for familiar stimuli (Bornstein, 1989; Zajonc, 1968). In contrast, source memory for faces of cheatersthat is, memory for the cheating context in which a face was encountered - can be instrumental in avoiding cheaters and, thus, should be beneficial to socially cooperating individuals.

Consistent with these considerations, a series of experiments in our lab (Buchner, Bell, Mehl, \& Musch, 2009; see Figure 1 for an illustration of the results) showed that source memory, but not old-new discrimination, is enhanced for faces of cheaters (for related results, see Chiappe et al., 2004). This is consistent with a functional perspective on human memory, in that information that is important from a fitness perspective is especially well remembered. It has been previously suggested that memory advantages for faces of cheaters can be ascribed to a cognitive module that was shaped by natural selection to

R.Bell, raoul.bell@uni-duesseldorf.de 

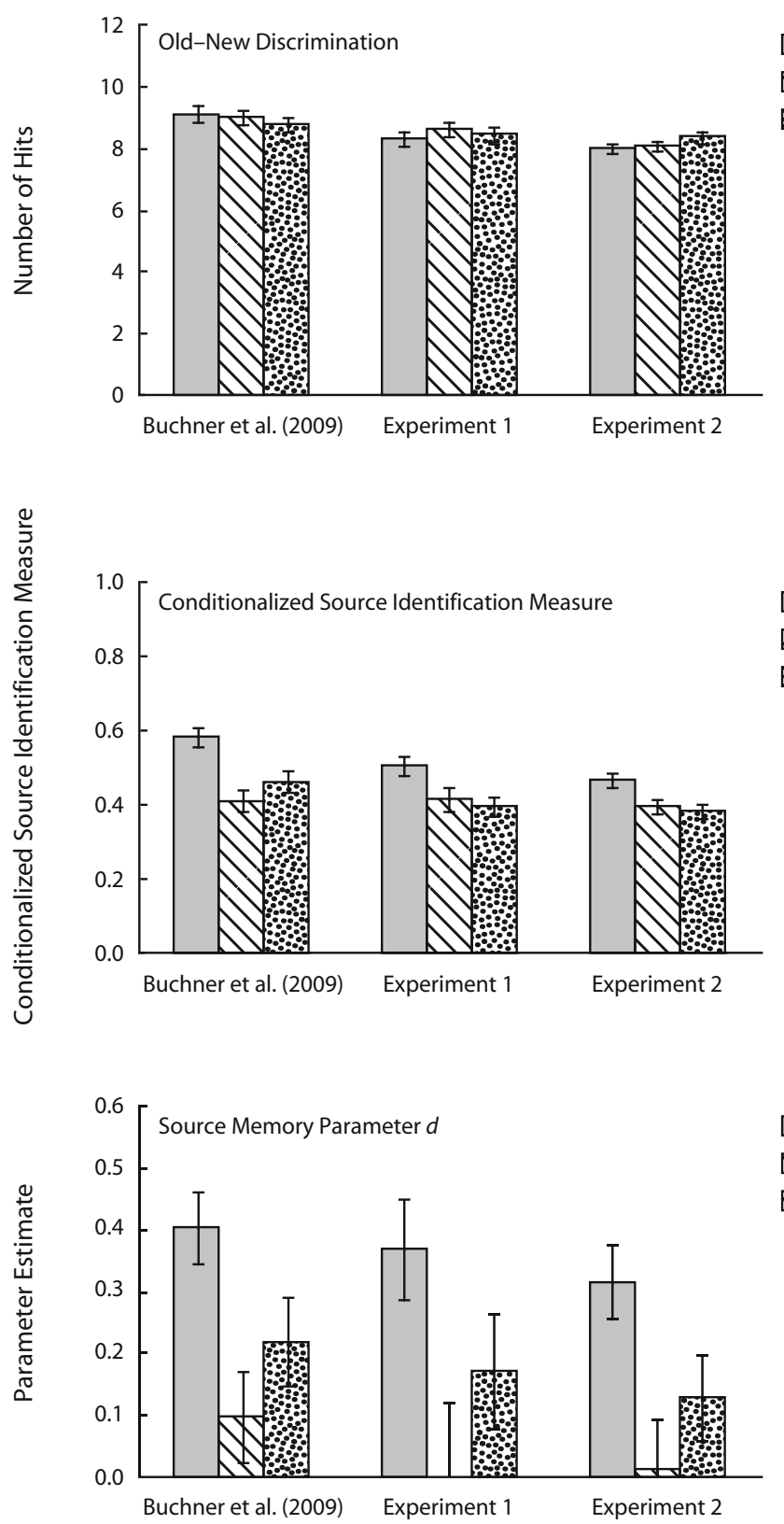

Figure 1. Memory measures as a function of valence. "Negative" and "positive" refer to disgusting and pleasant descriptions in the present experiments. For comparison, the results of Buchner, Bell, Mehl, and Musch's (2009) Experiment 2 are also presented. That experiment was identical to Experiment 1 reported here, except that descriptions of cheating were used as negative stimulus material, descriptions of trustworthy behavior were used as positive stimulus material, and a different set of facial photographs was used. Upper panel: Test phase old-new discrimination in terms of hits (12 at most). Error bars represent the standard errors of the means. The mean false alarm rate was .12 $(S D=.11)$ in Experiment 1 and $.14(S D=.10)$ in Experiment 2. Center panel: Correct source classifications conditionalized on the number of old judgments. Error bars represent the standard errors of the means. Lower panel: Parameter estimates for the source memory parameters for faces associated with negative information $\left(d_{-}\right.$in Figure 2$)$, for faces associated with neutral information ( $d_{\sim}$ in Figure 2), and for faces associated with positive information $\left(d_{+}\right.$in Figure 2). Error bars represent the .95 confidence intervals. 
"solve" the adaptive problem of avoiding exploitation in social exchange (Chiappe et al., 2004; Mealey et al., 1996; Oda, 1997). This explanation implies that the underlying mechanism is content specific, in that it is triggered only by intentional violations of social-exchange rules. However, this strong assumption should not be accepted without first considering the theoretically more parsimonious assumption that the underlying mechanism may be more general than that. Whether the source memory advantage for faces of cheaters is really due to highly specialized, content-specific memory processes is ultimately an empirical question.

For instance, one might claim that cheating is remembered better simply because it violates social norms and can therefore be considered exceptional and, thus, more distinct and memorable (Schmidt, 1991) than trustworthy behavior, which is supposed to be the norm. A priori, this seems to be a plausible domain-general explanation for the cheater advantage in source memory, given that it has previously been observed that rarity within an experiment can modulate memory for cheaters (Barclay, 2008). This alternative explanation has already been examined in Buchner et al. (2009). In three of the four experiments reported in Buchner et al. (2009), the degree to which the behavior was exceptional was manipulated. In all the experiments, source memory for faces of cheaters was better than source memory for other faces, but memory was not affected by whether the behavioral descriptions were exceptional or ordinary.

Having excluded this alternative explanation, we now turn to the question of whether the source memory advantage for faces of cheaters may be due to more general effects of valence on cognitive processing. It has often been observed that human memory and attention have a negativity or threat bias (Buchner, Rothermund, Wentura, \& Mehl, 2004; De Bruin \& Van Lange, 1999; Öhman, Lundqvist, \& Esteves, 2001; Peeters \& Czapinski, 1990; Pratto \& John, 1991; Wentura, Rothermund, \& Bak, 2000). For instance, negative information-especially, threatening information - attracts attention more readily than does neutral and positive information (e.g., Buchner, Mehl, Rothermund, \& Wentura, 2006; Buchner et al., 2004; Pratto \& John, 1991; Wentura et al., 2000). Faces displaying negative emotions such as anger are detected more reliably and remembered better than happy faces (Horstmann \& Bauland, 2006; Öhman et al., 2001). In the social domain, negative information has more influence on judgments about a person's character than does positive information (De Bruin \& Van Lange, 1999; Peeters \& Czapinski, 1990). Baumeister, Bratslavsky, Finkenauer, and Vohs (2001) performed an extensive literature review to identify the effects of valence on cognitive processing and concluded that "it appears to be a basic, pervasive fact of psychology that bad is stronger than good" and that "a specific or mid-level theory may not be broad enough to account for it" (p. 360).

The finding that source memory for faces of cheaters is enhanced, whereas old-new face discrimination is less affected by whether a face was associated to cheating, trustworthy, or neutral contexts (Buchner et al., 2009), fits with other findings from the memory literature that suggest that recollection is enhanced by emotional valence of stimulus material, whereas familiarity-based memory processes are less affected by valence. For example, in the remember-know paradigm, remember responses have more frequently been assigned to negative items than were neutral (Kensinger \& Corkin, 2003) or positive (Ochsner, 2000) items. Know responses, in contrast, have not varied as a function of affective content (Kensinger \& Corkin, 2003; Ochsner, 2000). Furthermore, consistent with the results of Buchner et al. (2009), Doerksen and Shimamura (2001) found that free recall and source memory for the font color in which a word was presented were enhanced for emotional, in comparison with neutral, words, whereas old-new discrimination was not affected by valence (see also D'Argembeau \& Van der Linden, 2004). There are also a number of other studies that are consistent with the assumption that source memory is enhanced for emotional material (Mather \& Nesmith, 2008; Smith, Dolan, \& Rugg, 2004) and especially for negative material (Kensinger \& Corkin, 2003; Kensinger, O’Brien, Swanberg, Garoff-Eaton, \& Schacter, 2007).

However, despite the strong claims that valence generally enhances information processing (Baumeister et al., 2001), there are also a number of studies that suggest that source memory is not affected by emotional valence (Kensinger \& Schacter, 2006) and can even be impaired for emotional or negatively valent material (Anderson \& Shimamura, 2005; Cook, Hicks, \& Marsh, 2007; Mather et al., 2006; Rossmann, 1984). For example, Cook and colleagues showed that source memory for the modality in which a word was presented (visual or auditory) was worse for negative than for neutral words. On the basis of these and other results (e.g., Johnson, Nolde, \& De Leonardis, 1996; Kensinger, Piguet, Krendl, \& Corkin, 2005), it has been suggested that the presentation of an emotional stimulus can cause a narrowing of attention. Emotional valence may draw attention to the valent stimulus, thereby disrupting the binding of the stimulus to emotionally neutral background information. In consequence, memory for the valent stimulus content may benefit at the expense of memory for the stimulus-context association (e.g., Johnson et al., 1996; Mather et al., 2006).

To sum up, the literature regarding emotional valence and source memory is quite inconsistent. In an attempt to resolve the conflicting findings, it has been suggested that emotional valence enhances the binding of details of the learning episode that are central to the emotional experience but impairs the strength of the association between the emotional event and background information that is irrelevant to the emotional meaning of the event (Kensinger, 2007; Mather, 2007). According to these considerations, an important difference between the study of Buchner et al. (2009) and most of the previous studies in which valence and source memory have been examined is that Buchner et al. (2009) assessed source memory for valent contexts, rather than for contexts that were irrelevant to the valence dimension (e.g., location or font color; but see Anderson \& Shimamura, 2005; Smith et al., 2004). If source memory is generally enhanced for valent contexts 
that are central to the emotional relevance of the learning episode, we may expect to find better source memory for faces associated with negative behavior, regardless of the specific nature of the behavior.

Thus, the source memory advantage for cheaters that was observed by Buchner et al. (2009) may be due to a module specifically designed for the detection of cheaters in social-exchange situations. An alternative possibility is that the source memory advantage for faces of cheaters is due to a general source memory advantage for threatening contexts. The purpose of the present experiments was to see whether the source memory advantage would generalize to faces associated with other threatening contexts that do not imply violation of or adherence to social-exchange rules. For this purpose, descriptions of cheating were replaced by descriptions of negative behaviors from a different domain - namely, disgusting behaviors. Disgust is a strong negative emotion that serves to reduce the risk of infections by motivating avoidance of contaminants (e.g., body secretions, vermin, spoiled food) and individuals or objects that have come in contact with these contaminants (Curtis, Aunger, \& Rabie, 2004; Fessler, Eng, \& Navarrete, 2005; Rozin \& Fallon, 1987; Toronchuk \& Ellis, 2007). If we were to find the same pattern as that in Buchner et al. (2009) — that is, better source memory for threatening contexts - this finding would suggest that the source memory advantage for cheaters may not be due to a specialized cheater identification module designed to avoid exploitation in social exchange and that a domaingeneral explanation of the results is more appropriate.

\section{Measuring Source Memory}

A problem with measuring source memory is which measurement tool to use. Early approaches relied on ad hoc measures of source memory, which are problematic because they confound source memory with old-new discrimination and guessing processes (Bröder \& Meiser, 2007; Murnane \& Bayen, 1998). Fortunately, alternative measurement tools exist in terms of multinomial models of source memory (Batchelder \& Riefer, 1990; Bayen, Murnane, \& Erdfelder, 1996), which allow us to decompose raw source classification data into old-new discrimination, source memory, and various forms of guessing biases. We used the multinomial source-monitoring model developed and successfully validated by Bayen et al. (1996), which has been successfully applied in a number of experiments (e.g., Bayen, Nakamura, Dupuis, \& Yang, 2000; Bell, Buchner, \& Mund, 2008; D’Argembeau \& Van der Linden, 2004; Doerksen \& Shimamura, 2001; Spaniol \& Bayen, 2002). An adaptation of the model for the present purposes is presented in Figure 2.

The model displayed in Figure 2 contains 12 parameters, each of which represents the probability of a certain cognitive process. Parameter $D_{-}$represents the probability of recognizing a face associated to negative contexts (disgusting behavior) as old. Parameter $d_{-}$represents the conditional probability of remembering correctly that a recognized face was encountered in a negative context. If the source of a correctly recognized face is not known (which occurs with a probability of $1-d_{-}$), the correct source may still be guessed with a probability of $a_{+} \cdot a_{-}$. Alternatively, it may be incorrectly guessed that the face was associated to a positive context (pleasant behavior) with a probability of $a_{ \pm} \cdot\left(1-a_{-}\right)$or to a neutral context with a probability of $\left(1-a_{ \pm}\right)$. If a face associated to a negative context is not recognized as old (with a probability of $1-D_{-}$), it may still be guessed, with a probability of $b$, that the face is old. For these faces, the negative source may be guessed with a probability of $g_{ \pm} \cdot g_{-}$. Alternatively, it may be incorrectly guessed that the face was associated to a pleasant context with a probability of $g_{ \pm} \cdot\left(1-g_{-}\right)$or to a neutral context with a probability of $\left(1-g_{ \pm}\right)$. Faces that are neither recognized as old (with a probability of $1-D_{-}$) nor guessed to be old (with a probability of $1-b$ ) are incorrectly judged to be new. Analogous considerations hold for the model trees for faces associated to neutral contexts, for faces associated to pleasant contexts, and for new faces.

Standard computer programs (e.g., Rothkegel, 1999; Stahl \& Klauer, 2007) can be used to estimate the probabilities associated with the model parameters representing the processes that lead to the source classifications. What is more, statistical tests can be performed directly on these model parameters. For instance, the hypothesis that source memory is better for negative than for positive contexts implies that the parameter representing source memory for negative contexts, $d_{-}$, is larger than the parameter representing source memory for positive contexts, $d_{+}$.

\section{EXPERIMENT 1}

Experiment 1 is an exact replication of the Experiment 2 reported by Buchner et al. (2009), with the only exception being that descriptions of disgusting, neutral, and pleasant behavior were used, instead of descriptions of cheating, neutral, and trustworthy behavior.

\section{Method}

Participants. The participants were 46 female and 15 male persons, most of whom were students at the Heinrich-Heine-Universität Düsseldorf. They were paid for participating. Their ages ranged from 16 to 44 years $(M=24, S D=5)$.

Apparatus and Materials. A total of 72 facial photographs of males (256-bit, $116 \times 164$ pixel grayscales) were randomly assigned to two sets of 36 photographs each (henceforth, Sets 1 and 2). The faces were taken from the Psychological Image Collection at Stirling (http://pics.psych.stir.ac.uk). Brief descriptions typed below the photographs conveyed either negative (disgusting), positive (pleasant), or neutral (neither disgusting nor pleasant) information about the person shown. As in earlier studies (Buchner et al., 2009; Mealey et al., 1996; Mehl \& Buchner, 2008), the descriptions also included information about the depicted person's profession, to indicate the person's social status. Status did not modulate the source memory advantage in Buchner et al. (2009). Therefore, it seemed justified to use only low-status descriptions to keep the design simple. The lowstatus job titles were the same as those used in Experiments 1-3 of Buchner et al. (2009). For instance, the following description would convey disgusting information: "K.S. is a laborer. To save money, he cooks dog food in a big pot to eat it all by himself." Care was taken to ensure that the disgusting descriptions did not imply direct harm to third parties, to accentuate the difference between the disgusting descriptions used here and the descriptions of cheating used in the previous studies. The stimulus material covered different types of 

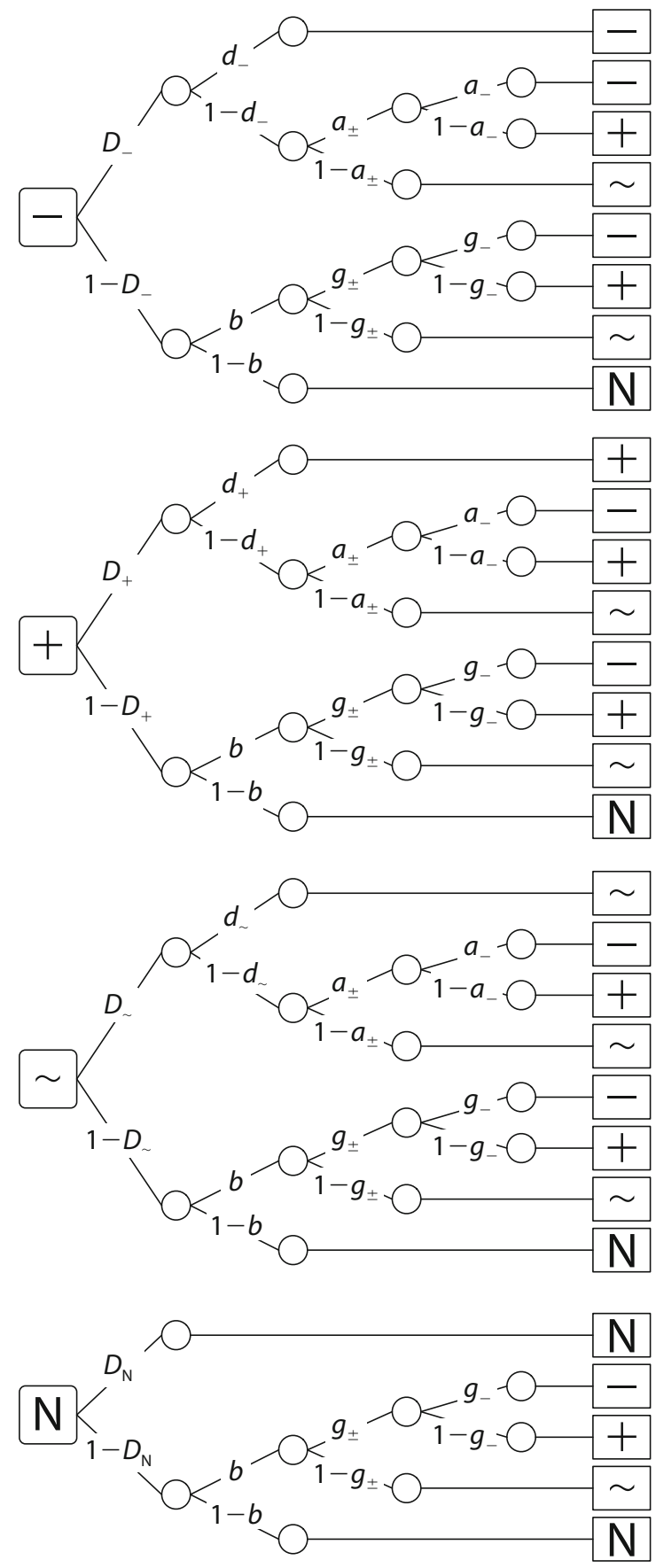

Figure 2. Bayen, Murnane, and Erdfelder's (1996) source memory model as adapted for the present purposes. Rounded rectangles on the left side represent the types of faces presented (faces associated with negative, neutral, and positive information are denoted by ,$- \sim$, and + , respectively; $\mathbf{N}$ denotes new faces not presented in the encoding phase of the experiment). Letters along the links represent the probabilities with which certain cognitive states occur $(D$, probability of correctly identifying a face as old [for previously presented faces] or new [for new faces]; $d$, source memory in the sense of remembering the context of encountering a face that was detected as old; $b$, probability of guessing that a nonrecognized face is old; $a_{ \pm}$, probability of guessing that a recognized face was encountered in a valenced context $a_{-}$, probability of guessing that a recognized face was encountered in a negative context; $g_{ \pm}$, probability of guessing that a nonrecognized face that was guessed to be old was encountered in a valenced context; $g_{-}$, probability of guessing that a nonrecognized face that was guessed to be old was encountered in a negative context). Rectangles on the right side represent the categories of participants' judgments (faces judged as having been associated with negative, neutral, and positive information are denoted by,$- \sim$, and + , respectively; $\mathbf{N}$ denotes faces judged to be new faces not presented in the encoding phase of the experiment). 
disgusting information, including lack of hygiene (e.g., "wipes his nose with his shirt sleeve"), intake of spoiled food (e.g., "uses dirty, musty pots"), body secretions (e.g., "urinates in the bathtub"), animals (e.g., "lets the dog lick his face"), and injuries (e.g., "suffered a serious burn injury that does not heal"). The following description would convey behavior that is neutral (neither disgusting nor pleasant): "J.L. is a gardener. He often orders lunch at work from a local Italian restaurant, because he cannot cook very well." The following description would convey pleasant behavior: "O.H. is a miller. When he has friends over, the smell of freshly baked cakes and cookies fills his apartment." In German, all the sentences were 20 words long, not including the two initial letters representing the person's fictitious names.

Information about the social status of the profession and the valence of the behavior descriptions was obtained in independent norming studies. In one norming study, participants $(N=36)$ rated 200 job titles with respect to their social status, using a scale ranging from 1 (low status) to 5 (high status). A total of 36 job titles with low ratings were used for the experiment $(M=1.88, S D=0.33)$. A different group of participants $(N=45)$ rated the valence of 50 behavior descriptions to make sure that instances of disgusting, neutral, and pleasant behavior were perceived as negative, neutral, and positive, respectively. Valence was assessed on a scale ranging from -3 (negative) to +3 (positive). Twelve sentences were selected for each of the three different sets of descriptions corresponding to the three levels of the behavioral history variable (negative, neutral, or positive). Mean valence was -1.85 for the disgusting descriptions $(S D=1.04), 0.27$ for the neutral descriptions $(S D=0.84)$, and 1.74 for the pleasant descriptions $(S D=0.95)$. In terms of absolute valence (i.e., ignoring the minus sign for the disgusting descriptions), an item-based analysis showed that there was a large difference between disgusting and neutral descriptions $[t(22)=22.50$, $p<.001]$ and between pleasant and neutral descriptions $[t(22)=$ $35.43, p<.001]$, whereas disgusting and pleasant descriptions did not differ $[t(22)=1.36, p=.19]$. The photographs and descriptions were combined randomly for each participant.

Procedure. The participants were tested individually. They were asked to rate the likability of 36 (Set 1 or 2, counterbalanced across participants) facial photographs that were presented in random order during the encoding phase. Each trial started with a headline ("How likable do you find this person?") and a photograph. The behavior description was shown $2 \mathrm{sec}$ later, followed $4.5 \mathrm{sec}$ later by the likability rating scale (ranging from 1, not likable at all, to 6, extremely likable). The participants rated the likability using the computer mouse and then initiated the next trial. The behavioral descriptions were randomly assigned to the faces.

As in Mehl and Buchner's (2008) Experiment 3 and Buchner et al.'s (2009) Experiments 1, 2, and 4, the encoding phase was directly followed by the test phase. Here, the participants saw a random sequence of 72 faces, half of which had been presented before (Set 1 or 2, depending on the encoding phase assignment) and half of which were new (Set 2 or 1). Each trial started with a headline ("How likable do you find this person?") and a photograph. The likability rating scale appeared $1.5 \mathrm{sec}$ later. After the rating, a new headline appeared ("Is this face old or new?"), followed by an "old" and a "new" checkbox, one of which the participants selected to indicate that they had seen a face during the encoding phase or not. Following an old judgment and a click on the continue button, checkboxes labeled "disgusting," "pleasant," and "neither disgusting nor pleasant" appeared, which the participants used to judge the behavior that was used in the description accompanying that face in the encoding phase. Selecting one of these checkboxes and then clicking the continue button initiated the next trial.

Design. The within-subjects independent variable was behavioral history (negative, neutral, positive). The dependent measures were likability ratings, old-new discrimination in terms of hits (given that there was only one set of new faces in the test phase, there was only one false alarm rate for all types of faces, so that sensitivity measures would be redundant), and source judgments for an old judgment.

Given a sample size of $N=61, \alpha=.05$, and the assumption that the average population correlation between the levels of the behavioral history repeated measures variable is $\rho=.55$ (estimated from pilot data), effects of size $f=0.20$ (Cohen, 1988) of this variable on old-new discrimination and the likability ratings could be detected with a probability of $1-\beta=.95$. All power calculations reported in this article were conducted using G*Power (Faul, Erdfelder, Lang, $\&$ Buchner, 2007). A multivariate approach was used for all withinsubjects comparisons. In the present application, all multivariate test criteria correspond to the same (exact) $F$ statistic, which is reported. Partial $\eta^{2}$ is reported as a measure of the size of an effect. The level of $\alpha$ was set to .05 , except for post hoc tests, for which the significance level was Bonferroni-Holm corrected (Holm, 1979).

\section{Results}

Encoding phase likability ratings. A repeated measures MANOVA showed that behavioral history had large effects on the encoding phase likability ratings [see Table 1; $\left.F(2,59)=112.12, p<.001, \eta_{\mathrm{p}}^{2}=.79\right]$. Orthogonal contrasts showed that faces associated with negative contexts were less likable than the other two types of faces $[F(1,60)=$ $\left.225.71, p<.001, \eta_{\mathrm{p}}^{2}=.79\right]$. Faces associated with neutral contexts were less likable than faces associated with positive contexts $\left[F(1,60)=11.33, p=.001, \eta_{\mathrm{p}}^{2}=.16\right]$.

Old-new discrimination. A repeated measures MANOVA showed that behavioral history did not affect old-new discrimination in terms of the number of hits [see Figure $\left.1 ; F(2,59)=0.65, p=.53, \eta_{\mathrm{p}}^{2}=.02\right]$. Thus, replicating earlier studies that used descriptions of cheating, trustworthy, and neutral behavior (Buchner et al., 2009; Mehl \& Buchner, 2008), despite the powerful effects of the descriptions on the encoding phase likability ratings, there was no effect of these descriptions on old-new discrimination.

Source memory. Given these results, it is now interesting to look at participants' source memory - that is, their memory for the affective context in which a face was encountered. In a first step, it may be helpful to look at the number of correct source classifications conditionalized on the number of items given an old classification (Figure 1). Conditionalized source classifications were more accurate for faces associated with disgusting descriptions than for other faces $\left[F(1,60)=10.48, p<.01, \eta_{\mathrm{p}}^{2}=.15\right]$. Unfortunately, as we have seen in the Measuring Source

Table 1

Encoding Phase and Test Phase Likability Ratings in Experiments 1 and 2 (Sample Means With Standard Errors of the Means)

Negative Neutral Pleasant

Context Context Context

$\begin{array}{llllll}M & S E & M & S E & M & S E\end{array}$

Encoding Phase Likability Ratings

$\begin{array}{lllllll}\text { Experiment 1 } & 2.15 & 0.08 & 3.52 & 0.08 & 3.74 & 0.10\end{array}$

$\begin{array}{lllllll}\text { Experiment } 2 & 2.21 & 0.06 & 3.41 & 0.05 & 3.74 & 0.06\end{array}$

Test Phase Likability Ratings

\begin{tabular}{lllllll} 
Experiment 1 & 2.87 & 0.07 & 3.07 & 0.08 & 3.11 & 0.08 \\
\hline
\end{tabular}

\begin{tabular}{lllllll} 
Experiment 2 & 2.94 & 0.05 & 3.07 & 0.05 & 3.07 & 0.05 \\
\hline
\end{tabular}


Memory section, correct classifications of faces associated with disgusting descriptions can be arrived at on the basis of many different types of processes. We thus need to decompose classification performance into the processes involved by using the multinomial model illustrated in Figure 2 to see whether source memory for faces associated to disgusting descriptions is really better than source memory for other types of faces.

In order to simplify our analysis, we began with a base model that builds on the fact that the old-new discrimination judgments did not differ as a function of behavioral history. We thus decided for the base model to set all parameters representing old-new discrimination for the different types of faces to be equal. We also set the parameter representing the probability of detecting new faces as new to be equal to the old-new discrimination parameters. This is the standard assumption of the twohigh threshold model of signal detection (Snodgrass \& Corwin, 1988) and is empirically justified by the wellknown mirror effect (Glanzer, Adams, Iverson, \& Kim, 1993). As a consequence, the base model is characterized by the general restriction that $D_{-}=D_{\sim}=D_{+}=D_{\mathrm{N}}$. As can be seen in Table 2, the base model fits the data extremely well. This finding further supports the hypothesis that old-new discrimination of the faces was independent of whether they were associated with negative, neutral, or positive contexts.

The remaining hypothesis tests will build on the base model. First, we tested the central hypothesis that source memory is better for faces encountered in a negative context than for faces encountered in a positive context. Descriptively, this appears to be the case (Figure 1; the full set of parameter estimates is reported in the Appendix). The null hypothesis of no such difference is tested by imposing, on the base model, the restriction that $d_{-}=d_{+}$ where $d_{-}$and $d_{+}$represent source memory for negative (disgusting) and positive (pleasant) contexts, respectively. This restriction generates one additional degree of freedom. The corresponding increase in the goodness-of-fit statistic $G^{2}$ over that of the base model is asymptotically $\chi^{2}$ distributed with one degree of freedom. The second row of Table 2 shows that the restriction $d_{-}=d_{+}$was clearly not compatible with the data. We must reject the hypothesis of no difference in source memory between negative and positive contexts and, instead, conclude that source memory for faces encountered in negative contexts is significantly better than source memory for faces encountered in positive contexts.
Second, we also tested whether the descriptive difference between parameters $d_{+}$and $d_{\sim}$ was statistically significant. The statistical test of this hypothesis is parallel to the one just described. The result displayed in the third row of Table 2 shows that this difference was statistically significant. In other words, source memory for faces associated with pleasant behavior was somewhat better than source memory associated with neutral behavior, although the difference in source memory between negative and positive contexts was much larger than the difference in source memory between positive and neutral contexts. An equally small but significant difference between source memory for faces associated with trustworthy and neutral behaviors was observed in Experiment 2 of Buchner et al. (2009).

It is interesting that the results of the analysis of the conditionalized source classifications differ from the multinomial modeling results in that the latter reveal a significant difference between the positive and the neutral conditions that is not present in the conditionalized source classifications. The reason for this discrepancy is most likely that the participants were more prone to guess that they had encountered a face in a neutral context than in a positive context, especially when the face was not recognized [the probability of guessing that a face was encountered in a neutral context was $\left(1-a_{ \pm}\right)=.40$ for recognized faces and $\left(1-g_{ \pm}\right)=.49$ for nonrecognized faces, whereas the probability of guessing that a face was encountered in a positive context was $a_{ \pm} \cdot\left(1-a_{-}\right)=$ .32 and $g_{ \pm} \cdot\left(1-g_{-}\right)=.23$, respectively]. Thus, this result highlights the importance of using a source memory measure that takes guessing into account (for more detailed discussions, see Bröder \& Meiser, 2007; Murnane \& Bayen, 1998).

Test phase likability ratings. A supplementary analysis of the test phase likability ratings (Table 1) showed a significant main effect of behavioral history $[F(2,59)=$ $\left.11.92, p<.001, \eta_{\mathrm{p}}^{2}=.29\right]$. Faces associated with negative contexts were less likable than other faces $[F(1,60)=$ $\left.22.78, p<.001, \eta_{\mathrm{p}}^{2}=.28\right]$, whereas likability ratings did not differ between faces associated with neutral and pleasant descriptions $\left[F(1,60)=0.10, p=.43, \eta_{\mathrm{p}}^{2}=.01\right]$.

\section{Discussion}

The results of Experiment 1 replicated those of Buchner et al. (2009) in showing that despite the large effects of the behavioral descriptions on encoding phase likability ratings, old-new face discrimination was unaffected by

Table 2

Model-Based Results of Source Memory

\begin{tabular}{lll}
\hline \multicolumn{1}{c}{ Model Test (Parameter Restriction) } & \multicolumn{1}{c}{ Experiment 1} & \multicolumn{1}{c}{ Experiment 2} \\
\hline Base model: $\left(D_{-}=D_{\sim}=D_{+}=D_{\mathrm{N}}\right)$ & $G^{2}(3)=2.40, p=.49$ & $G^{2}(3)=6.22, p=.10$ \\
Additional restriction on base model: $d_{-}=d_{+}$ & $\Delta G^{2}(1)=9.10, \boldsymbol{p}<. \mathbf{0 1}$ & $\Delta G^{2}(1)=14.90, \boldsymbol{p}<. \mathbf{0 0 1}$ \\
Additional restriction on base model: $d_{+}=d_{\sim}$ & $\Delta G^{2}(1)=5.53, \boldsymbol{p}=. \mathbf{0 2}$ & $\Delta G^{2}(1)=3.60, p=.06$ \\
\hline
\end{tabular}

Note-The goodness-of-fit of the base models and the additional restrictions were tested using the goodness-of-fit statistic $G^{2}$, which is asymptotically $\chi^{2}$ distributed with degrees of freedom indicated in parentheses. The $p$ values smaller than .05 (printed in bold) indicate that the implemented restrictions are not compatible with the data, as a result of which the hypothesis implied by the parameter restriction must be rejected. 
whether the faces were originally associated with negative, positive, or neutral contexts. In contrast, source memory was clearly modulated by the valence of the behavioral descriptions. Source memory for faces encountered in disgusting contexts was increased, in comparison with source memory for faces encountered in neutral and pleasant contexts. Source memory for faces encountered in pleasant contexts was somewhat better than source memory for faces associated with neutral contexts, but only slightly so. Test phase likability ratings were worse for faces associated with disgusting contexts than for other types of faces, whereas test phase likability ratings did not differ between pleasant and neutral contexts. This may reflect the participants' memory for the disgusting descriptions associated with the faces, which may translate into a negative reaction toward that person.

In sum, all of the results that were obtained by Buchner et al. (2009) using descriptions of cheating, trustworthy, and neutral behavior were replicated in the present Experiment 1 . This suggests that the source memory advantage for the faces of cheaters may not be due to a highly specialized module designed for the detection of cheaters in social exchange situations but, rather, to more general effects of valence on memory.

However, given the fact that the literature concerning emotional valence and source memory is highly inconsistent and several results have been difficult to replicate (see the introduction), we thought it important to demonstrate the robustness of the effects of Experiment 1 before drawing final conclusions. In addition to replicating the results of Experiment 1, Experiment 2 was designed to address two further questions. First, we wanted to know whether the source memory advantage for disgusting contexts depends on the personal predispositions of the participants. More precisely, we wanted to know whether interindividual differences in disgust sensitivity would modulate the effects observed in Experiment 1. Given that the effect of the behavioral history on source memory seems to rely on emotional reactions of the participants to the behavioral descriptions, disgust-sensitive individuals may show more pronounced effects of the behavioral history than disgust-insensitive individuals. Furthermore, it has previously been shown that participants look longer at faces associated with negative contexts (cheating descriptions) than at other faces when asked to rate the attractiveness of the faces (Chiappe et al., 2004). Thus, participants may remember faces associated with negative contexts better than faces associated with positive contexts simply because they look longer at the faces associated with negative contexts. To examine whether encoding time is essential in explaining the negativity bias in source memory, the encoding time of the faces was either fixed or variable in Experiment 2.

\section{EXPERIMENT 2}

\section{Method}

Participants. The participants were 91 female and 44 male persons who were paid for participating. Most of them were students at the Heinrich-Heine-Universität Düsseldorf. Their ages ranged from
18 to 56 years $(M=24, S D=6)$. They had not participated in Experiment 1.

Apparatus, Materials, Procedure, and Design. The apparatus, materials, procedure, and design were identical to those in Experiment 1, with the following exceptions. After the experiment, the participants were required to fill out a questionnaire to assess their disgust sensitivity (Schienle, Walter, Stark, \& Vaitl, 2002). The questionnaire consisted of five scales-(1) death, (2) body secretions, (3) hygiene, (4) spoilage, and (5) oral rejection - and had a reliability (Cronbach's $\alpha$ ) of .90. In order to test whether responding to this questionnaire was in some way affected by having been exposed to items associated with disgust, we compared the overall questionnaire scores of the participants of Experiment $2(N=138)$ with the scores of a different sample of $N=70$ participants who had filled out the same questionnaire after an unrelated attention experiment. The difference between groups was not significant $[F(1,204)=1.40, p=$ $\left..24, \eta_{\mathrm{p}}^{2}=.01\right]$; thus, we concluded that responding to the disgust sensitivity questionnaire was not affected by having been exposed to disgust-related information during the experiment.

The participants were assigned to two experimental groups. The participants in the self-paced group were allowed to choose how long they looked at a particular face before judging the likability of the stimulus person. After the face and the behavioral description were presented, a button with the label "rating" appeared. The participants looked at the face and the behavioral description as long as they wanted. After clicking the button, the face and the behavioral description disappeared, and the likability-rating scale was shown. It was assessed how long the participants had seen the face. In the experimenter-paced group, both the face and the behavioral description disappeared after $8,000 \mathrm{msec}$ and the likability-rating scale was shown.

Given $N=135, \alpha=.05$, and $\rho=.55$, effects of size $f=.13$ of the behavioral history variable on old-new discrimination and the likability ratings could be detected with a probability of $1-\beta=.95$.

\section{Results}

Encoding phase viewing time and likability ratings. Our expectation that the participants in the self-paced group would look longer at the faces associated with negative contexts than at the faces associated with positive or neutral contexts was not confirmed by the analysis of the encoding phase viewing time. For the self-paced group, viewing time was $8,273 \mathrm{msec}$ for the faces associated with disgusting descriptions $(S E=255), 8,327 \mathrm{msec}$ for the faces associated with neutral descriptions $(S E=242)$, and $8,183 \mathrm{msec}$ for the faces associated with pleasant descriptions $(S E=270)$. Behavioral history had no significant effect on viewing time in the self-paced group $[F(2,66)=$ $\left.0.78, p=.46, \eta_{\mathrm{p}}^{2}=.02\right]$. Encoding time also did not differ between the self-paced group and the experimenter-paced group $\left[F(1,133)=1.12, p=.29, \eta_{\mathrm{p}}^{2}<.01\right]$. Therefore, we dropped this variable from further analyses.

Encoding phase likability ratings (Table 1 ) differed as a function of behavioral history $[F(2,133)=203.51, p<$ $\left..001, \eta_{\mathrm{p}}^{2}=.75\right]$, and this effect was again very large. Faces associated with negative contexts were rated significantly less likable than other faces $[F(1,134)=409.94, p<.001$, $\left.\eta_{\mathrm{p}}^{2}=.75\right]$, and faces associated with neutral contexts were less likable than faces associated with positive contexts $\left[F(1,134)=14.40, p<.001, \eta_{\mathrm{p}}^{2}=.27\right]$.

Old-new discrimination. Old-new discrimination did not differ as a function of behavioral history $[F(2,133)=$ $\left.2.22, p=.11, \eta_{\mathrm{p}}^{2}=.03\right]$. This replicates the results of Experiment 1 and the results of previous experiments using 
descriptions of cheating, trustworthy, and neutral behavior (Buchner et al., 2009; Mehl \& Buchner, 2008).

Source memory. The conditionalized source classification scores (Figure 1) were again more accurate for faces associated with disgusting contexts than for the other types of faces $\left[F(1,134)=11.22, p<.01, \eta_{\mathrm{p}}^{2}=.08\right]$. Next, we need to decompose classification performance into the processes involved. The base model again fitted the data (Table 2). For the central hypothesis test, the second row of Table 2 shows that the $d_{-}=d_{+}$restriction was incompatible with the data. Given the positive difference between the estimates of $d_{-}$and of $d_{+}$(Figure 1; the full set of parameter estimates is reported in the Appendix), we conclude that source memory was better for faces associated to negative contexts than for faces associated to positive contexts. The third row of Table 2 shows that the descriptive difference between the parameters $d_{+}$and $d_{\sim}$ was not statistically significant. We thus have to conclude that source memory did not differ between faces associated with positive and neutral contexts. But note that the difference just misses statistical significance.

Test phase likability ratings. A supplementary analysis of the test phase likability ratings (Table 1 ) showed a significant main effect of behavioral history $[F(2,133)=$ $\left.5.80, p<.01, \eta_{\mathrm{p}}^{2}=.08\right]$. Faces associated with negative contexts were less likable than other faces $[F(1,134)=$ $11.61, p<.01, \eta_{\mathrm{p}}^{2}=.08$ ], whereas likability did not differ between faces associated with neutral contexts and faces associated with positive contexts $[F(1,134)=0.02, p=$ $\left..90, \eta_{\mathrm{p}}^{2}<.01\right]$.

Disgust sensitivity. General disgust sensitivity correlated with average encoding phase likability ratings of faces associated with disgusting contexts $(r=-.43, p<$ $.001)$, but not with average encoding phase likability ratings of other faces $(r \geq-.09, p \geq .28)$. Likewise, general disgust sensitivity correlated with average test phase likability ratings of faces associated with disgusting contexts $(r=-.24, p<.01)$, but not with average test phase likability ratings of other faces $(r \geq-.14, p \geq .11)$. Of the subscales, the "hygiene" scale had the highest correlation with likability scores in the disgusting context condition $(r=-.37, p<.001$, and $r=-.23, p<.01$, for encoding phase and test phase likability scores, respectively), probably reflecting the fact that most of the descriptions could be assigned to this category. Nevertheless, the correlations between the "hygiene" subscale and likability ratings were lower than those between general disgust sensitivity and likability ratings, which may have been due to the higher reliability of the complete disgust sensitivity scale, relative to that of one single subscale. We therefore decided to examine whether general disgust sensitivity would be related to source memory for faces associated with disgusting behavior. To this end, we assigned the participants to two groups on the basis of whether their general disgust sensitivity score was above or below sample median. Unfortunately, the model-based analysis does not allow for a direct test of the interaction of behavioral history and disgust sensitivity. We therefore need to rely on an indirect evaluation of this hypothesis by looking at the relative sizes of the disgust advantage in the two disgust sensitivity groups. The high-disgust-sensitivity group showed better source memory for faces associated with disgusting contexts $\left(d_{-}=.34\right)$ than for faces associated with pleasant contexts $\left(d_{+}=.11\right)$. The difference in source memory for these two types of faces was large and statistically significant even at the .001 level $\left[\Delta G^{2}(1)=11.22, p<.001\right.$, $w=.05]$. The low-disgust-sensitivity group also showed better source memory for faces associated with disgusting contexts $\left(d_{-}=.29\right)$ than for faces associated with pleasant contexts $\left(d_{+}=.15\right)$. The difference in source memory for these two types of faces was descriptively smaller than that observed in the high-disgust-sensitivity group, albeit it just reached the significance level $\left[\Delta G^{2}(1)=4.21, p<\right.$ $.04, w=.03]$. Thus, in terms of the standardized effect size measure $w$, the disgust advantage was about $40 \%$ larger in the high- than in the low-sensitivity group.

\section{Discussion}

The results of Experiment 2 replicated the results of Experiment 1. First, old-new discrimination was not affected by the behavioral descriptions. Second, both test phase likability ratings and source memory were affected by the behavioral history variable. Source memory for faces associated with negative contexts was better than source memory for faces associated with positive contexts, confirming the general preponderance of negative versus positive information for the present experiment. In other words, the results were again strikingly similar to the results found in experiments using behavioral descriptions of cheating, trustworthy, and neutral behavior.

This time, source memory for faces associated with positive contexts was not better than source memory for faces associated with neutral contexts. Given that there was a descriptive tendency toward better source memory for pleasant contexts than for neutral contexts that just fell short of statistical significance, the failure to replicate the small difference in source memory for positive and neutral contexts observed in Experiment 1 may well have been due to chance. Although the finding of no significant difference between source memory for positive and neutral contexts is inconsistent with the results of Experiment 2 in Buchner et al. (2009), it is consistent with the majority of results reported by Buchner et al. (2009), in which the difference in source memory between trustworthy and neutral contexts was statistically significant only in one of four experiments and when the data from all four experiments were combined. Parallel to these findings, the difference in source memory between pleasant and neutral contexts is clearly significant when the data from both Experiment 1 and Experiment 2 are combined $\left[\Delta G^{2}(1)=8.43, p<.01, w=.02\right]$, although it is still much smaller than the difference between disgusting and pleasant descriptions $\left[\Delta G^{2}(1)=23.78, p<.001, w=\right.$ .04]. Thus, whereas the difference in source memory between faces associated with negative contexts and faces associated with positive contexts is relatively large and, therefore, easy to replicate with moderate sample sizes, the difference in source memory between faces associated with positive contexts and faces associated with neutral contexts appears to be of smaller magnitude and is, there- 
fore, difficult to replicate unless extremely large samples are used.

The effects of the behavioral history on test phase likability ratings and source memory cannot be due to differences in encoding time, because behavioral history had no significant effects on viewing time in the self-paced group and, nevertheless, exerted effects on test phase likability ratings and source memory. The significant correlations between disgust sensitivity and the encoding phase and test phase likability ratings suggest that the emotional reaction to the behavioral descriptions was mediated by disgust sensitivity. The source memory advantage for faces associated with negative contexts was replicated in both the high- and the low-disgust-sensitivity groups, although the difference in source memory for disgusting and pleasant contexts was descriptively larger in the highdisgust-sensitivity group, in comparison with the lowdisgust-sensitivity group.

\section{GENERAL DISCUSSION}

In summary, Experiments 1 and 2 show that old-new face discrimination did not differ as a function of whether faces were associated with disgusting, pleasant, or neutral contexts, but source memory for faces associated with disgusting contexts was better than source memory for other types of faces. Given that the results closely replicate results obtained with descriptions of cheating, trustworthy, and neutral behaviors (Buchner et al., 2009), it is not necessary to interpret these results as being due to some special memory mechanisms that are confined to the particular type of emotion involved (disgust). Instead, it may be suggested that these results can be interpreted as being due to more general effects of valence on memory. We therefore conclude that source memory is increased for faces encountered in negative or threatening contexts, in comparison with faces encountered in neutral or positive contexts, whereas old-new face discrimination is not modulated by the emotional valence of the context a face was associated with.

Given the fact that the literature concerning valence and source memory is quite inconsistent, it seems notable that the results of six experiments (the two experiments of the present study and the four experiments reported in Buchner et al., 2009) consistently replicated the finding that source memory for negative sources is enhanced. Furthermore, these results are consistent with data showing that source memory is enhanced by valence and, especially, by negative valence (D'Argembeau \& Van der Linden, 2004; Doerksen \& Shimamura, 2001; Kensinger \& Corkin, 2003; Kensinger et al., 2007; Mather \& Nesmith, 2008; Smith et al., 2004). However, there are also a large number of studies showing that source memory is decreased by emotional or negatively valent material (Anderson \& Shimamura, 2005; Cook et al., 2007; Mather et al., 2006; Rossmann, 1984). As was noted earlier, a crucial difference between the present study and most studies showing decreased source memory for emotional items may be that, here, we assessed source memory for valent source information, whereas most other studies have assessed source memory for peripheral, nonemotional context information (but see Anderson \& Shimamura, 2005). Therefore, the present results seem largely consistent with the assumption that memory for context information that is central to the emotional relevance of an encoding episode is enhanced (cf. Kensinger, 2007; Kensinger et al., 2005; Mather, 2007).

Another important distinction between the present study and most previous studies is that remembering that someone was associated with disgust has obvious fitness advantages. If one assumes that memory is adaptive to the extent that it can guide our future behavior, it makes sense that we best remember emotionally valent context information that allows us to decide whether to approach or avoid a particular person. From a functional perspective, remembering the location or font color of an emotional word may be something very different from remembering the location of a predator, or whether a face belongs to a potentially dangerous person, even though all of these tasks would be classified as source memory tasks. One may expect that source memory advantages can be obtained more consistently for fitness-relevant contexts. In this sense, the present results support the general assumption that it may be fruitful to analyze human memory performance with respect to past (and present) fitness advantages (Nairne \& Pandeirada, 2008a, 2008b; Nairne et al., 2008; Nairne et al., 2007).

An interesting aspect of the present data, as well as those in Buchner et al. (2009), is that the source memory advantage for faces encountered in negative contexts was large and easy to replicate, whereas the source memory advantage for faces encountered in positive contexts (relative to faces associated with neutral contexts) was much smaller and significant in only a few cases. This data pattern fits with other findings in the memory literature showing similar positive-negative asymmetries in recollection and source memory (Kensinger et al., 2007; Ochsner, 2000).

The negativity bias was also present in the encoding phase and test phase likability ratings. Given the fact that the positively and negatively valent descriptions were selected because norming studies have shown that valence strength is similar for the two types of descriptions, the much stronger effect of the disgusting descriptions needs an explanation. The enhanced effects of negative over positive descriptions may be attributed to general negative-positive asymmetries in information processing. It is known that negatively valent information often attracts more attention than does positive information (Buchner et al., 2004; Pratto \& John, 1991; Wentura et al., 2000). Furthermore, it has been suggested that negative emotions lead to deeper, more detailed processing than do positive emotions (Bless et al., 1996; Bless \& Fiedler, 2006). The different ways in which negative and positive stimuli are processed may influence the affective reactions to these stimuli. For example, it has been shown that when negative and positive information is combined with other information, the negative information has more influence on evaluative processing than does positive or neutral information (Peeters \& Czapinski, 1990; 
Skowronski \& Carlston, 1989). This could explain why the negative behavioral descriptions that did not differ in valence strength from the positive descriptions in the norming study nevertheless exerted larger effects on source memory and likability ratings in the experimental context.

The greater sensitivity to negative than to positive stimuli may be adaptive because of a general asymmetry in the effects of positive and negative events on organisms. Negative events may generally have the potential to exert stronger and longer lasting effects on organisms than do positive events (Baumeister et al., 2001; Peeters \& Czapinski, 1990). It has also been noted that negative information about a person's behavior may be more informative about internal predispositions of that person than is positive information, because positive behavior can be more readily attributed to external sources such as social pressure (Rothbart \& Park, 1986; Skowronski \& Carlston, 1989). Therefore, negative information about a person may be more predictive of that person's future behavior than is positive information. In the present case, the adaptive benefit of remembering faces associated with disgusting behaviors would be to enable the individual to minimize the health risks that are associated with getting in contact with contaminants. Thus, being able to avoid persons associated with disgusting behaviors may be more directly related to survival and reproduction than being able to remember that a person was associated with neutral or pleasant behavior.

The present results replicate results obtained with descriptions of cheating, trustworthy, and neutral behaviors that were attributed to a cheater identification module confined to information from the social domain that has evolved to solve the adaptive problem of avoiding exploitation in social-exchange situations (Buchner et al., 2009). However, given that virtually the same results were observed regardless of whether faces were associated with cheating, trustworthy, or neutral behavior, the valence (or threat potential) of the descriptions, not their particular content, may determine effects on likability ratings and source memory. There is, however, an alternative to assuming a threat-sensitive encoding mechanism. One could postulate a cheater detection module to explain the finding of better source memory for cheater contexts reported by Buchner et al. (2009) and a disgust detection module to explain the findings of better source memory for disgust contexts reported here. In this way, one could assume more and more modules for every new valence dimension (aggression, fear, etc.) if it were shown that variations along these dimensions affected source memory. Although possible in principle, this theoretical alternative does not appear to be very attractive. First, from a purely theoretical point of view, it is much less parsimonious than assuming a general threat-sensitive encoding mechanism. Hence, the latter is to be preferred. Second, it seems plausible that the relative evolutionary benefit of one simple mechanism that serves the organism reasonably well in many different areas - such as a general threat-sensitive encoding mechanism - is much larger than the benefit of a multitude of independent yet functionally parallel mod- ules, each of which has been selected because it helped to solve the problem of avoiding negative influences in one particular subject area. In other words, the proposition of a threat-sensitive mechanism is consistent with an evolutionary point of view. Even Cosmides and Tooby (1992) consider the possibility that not all of the algorithms necessary for the maintenance of social cooperation need to be part of the same "mental organ." When these algorithms are "necessary to engage in social interactions other than exchange - such as aggressive threat - these might be activated even when the algorithms that are specific to social exchange are not" (p. 177).

To summarize, the present results suggest that the effects of behavioral descriptions on source memory that were previously ascribed to a highly specialized cheater identification module may, instead, be due to a more general negativity or threat bias in information processing. Negative valence may exert strong effects on source memory in the present experiments because it is directly related to the avoidance of potentially harmful interactions with dangerous individuals.

\section{AUTHOR NOTE}

The research reported in this article was supported by a grant from the Deutsche Forschungsgemeinschaft ( $\mathrm{Bu}$ 945/7-1). Correspondence concerning this article should be addressed to R. Bell or to A. Buchner, Institut für Experimentelle Psychologie, Heinrich-Heine-Universität, D-40225 Düsseldorf, Germany (e-mail: raoul.bell@uni-duesseldorf.de or axel.buchner@uni-duesseldorf.de).

\section{REFERENCES}

Anderson, L., \& Shimamura, A. P. (2005). Influences of emotion on context memory while viewing film clips. American Journal of Psychology, 118, 323-337.

Axelrod, R., \& Hamilton, W. D. (1981). The evolution of cooperation. Science, 211, 1390-1396.

BARCLAY, P. (2008). Enhanced recognition of defectors depends on their rarity. Cognition, 107, 817-828.

Barclay, P., \& Lalumière, M. L. (2006). Do people differentially remember cheaters? Human Nature, 17, 98-113.

BAtChelder, W. H., \& Riefer, D. M. (1990). Multinomial processing models of source monitoring. Psychological Review, 97, 548-564.

Baumeister, R. F., Bratslavsky, E., Finkenauer, C., \& Vohs, K. D. (2001). Bad is stronger than good. Review of General Psychology, 5, 323-370.

Bayen, U. J., Murnane, K., \& Erdfelder, E. (1996). Source discrimination, item detection, and multinomial models of source monitoring. Journal of Experimental Psychology: Learning, Memory, \& Cognition, 22, 197-215.

Bayen, U. J., Nakamura, G. V., Dupuis, S. E., \& Yang, C.-L. (2000). The use of schematic knowledge about sources in source monitoring. Memory \& Cognition, 28, 480-500.

Bell, R., Buchner, A., \& Mund, I. (2008). Age-related differences in irrelevant-speech effects. Psychology \& Aging, 23, 377-391.

Bless, H., Clore, G. L., Schwarz, N., Golisano, V., Rabe, C., \& Wolk, M. (1996). Mood and the use of scripts: Does a happy mood really lead to mindlessness? Journal of Personality \& Social Psychology, 71, 665-679.

Bless, H., \& FiedLer, K. (2006). Mood and the regulation of information processing and behavior. In J. P. Forgas (Ed.), Affect in social thinking and behavior (pp. 65-84). New York: Psychology Press.

Bornstein, R. F. (1989). Exposure and affect: Overview and metaanalysis of research, 1968-1987. Psychological Bulletin, 106, 265289.

BRÖDER, A., \& MEISER, T. (2007). Measuring source memory. Zeitschrift für Psychologie, 215, 52-60.

Buchner, A., Bell, R., Mehl, B., \& Musch, J. (2009). No enhanced 
recognition memory, but better source memory for faces of cheaters. Evolution \& Human Behavior, 30, 212-224.

Buchner, A., Mehl, B., Rothermund, K., \& Wentura, D. (2006). Artificially induced valence of distractor words increases the effects of irrelevant speech on serial recall. Memory \& Cognition, 34, 10551062.

Buchner, A., Rothermund, K., Wentura, D., \& Mehl, B. (2004). Valence of distractor words increases the effects of irrelevant speech on serial recall. Memory \& Cognition, 32, 722-731.

Carlisle, E., \& Shafir, E. (2005). Questioning the cheater-detection hypothesis: New studies with the selection task. Thinking \& Reasoning, 11, 97-122.

Chiappe, D., Brown, A., Dow, B., Koontz, J., Rodriguez, M., \& MCCulloch, K. (2004). Cheaters are looked at longer and remembered better than cooperators in social exchange situations. Evolutionary Psychology, 2, 108-120.

CoHEn, J. (1988). Statistical power analysis for the behavioral sciences. Hillsdale, NJ: Erlbaum.

CooK, G. I., Hicks, J. L., \& MARsh, R. L. (2007). Source monitoring is not always enhanced for valenced material. Memory \& Cognition, 35, 222-230.

Cosmides, L. (1989). The logic of social exchange: Has natural selection shaped how humans reason? Studies with the Wason selection task. Cognition, 31, 187-276.

Cosmides, L., \& Tooby, J. (1992). Cognitive adaptations for social exchange. In J. H. Barkow, L. Cosmides, \& J. Tooby (Eds.), The adapted mind: Evolutionary psychology and the generation of culture (pp. 163228). New York: Oxford University Press.

Cosmides, L., \& Toовy, J. (2005). Neurocognitive adaptations designed for social exchange. In D. M. Buss (Ed.), The handbook of evolutionary psychology (pp. 584-627). Hoboken, NJ: Wiley.

Curtis, V., Aunger, R., \& Rabie, T. (2004). Evidence that disgust evolved to protect from risk of disease. Proceedings of the Royal Society $B$, 271(Suppl. 4), S131-S133.

D'Argembeau, A., \& Van der Linden, M. (2004). Influence of affective meaning on memory for contextual information. Emotion, 4, 173-188.

De Bruin, E. N. M., \& VAN Lange, P. A. M. (1999). Impression formation and cooperative behavior. European Journal of Social Psychology, 29, 305-328.

Doerksen, S., \& Shimamura, A. P. (2001). Source memory enhancement for emotional words. Emotion, 1, 5-11.

Faul, F., Erdfelder, E., Lang, A.-G., \& Buchner, A. (2007). G*Power 3: A flexible statistical power analysis program for the social, behavioral, and biomedical sciences. Behavior Research Methods, 39, 175-191.

Fessler, D. M. T., EnG, S. J., \& Navarrete, C. D. (2005). Elevated disgust sensitivity in the first trimester of pregnancy: Evidence supporting the compensatory prophylaxis hypothesis. Evolution \& Human Behavior, 26, 344-351.

FidDick, L., \& RUTHERFord, M. D. (2006). Looking for loss in all the wrong places: Loss avoidance does not explain cheater detection. Evolution \& Human Behavior, 27, 417-432.

Glanzer, M., Adams, J. K., Iverson, G. J., \& Kim, K. (1993). The regularities of recognition memory. Psychological Review, 100, 546567.

HoLm, S. (1979). A simple sequentially rejective multiple test procedure. Scandinavian Journal of Statistics, 6, 65-70.

Horstmann, G., \& Bauland, A. (2006). Search asymmetries with real faces: Testing the anger-superiority effect. Emotion, 6, 193-207.

Johnson, M. K., Nolde, S. F., \& De Leonardis, D. M. (1996). Emotional focus and source monitoring. Journal of Memory \& Language, 35, 135-156.

Kensinger, E. A. (2007). Negative emotion enhances memory accuracy: Behavioral and neuroimaging evidence. Current Directions in Psychological Science, 16, 213-218.

Kensinger, E. A., \& Corkin, S. (2003). Memory enhancement for emotional words: Are emotional words more vividly remembered than neutral words? Memory \& Cognition, 31, 1169-1180.

Kensinger, E. A., O’Brien, J. L., Swanberg, K., Garoff-Eaton, R. J., \& SCHACTER, D. L. (2007). The effects of emotional content on reality-monitoring performance in young and older adults. Psychology \& Aging, 22, 752-764.
Kensinger, E. A., Piguet, O., Krendl, A. C., \& Corkin, S. (2005). Memory for contextual details: Effects of emotion and aging. Psychology \& Aging, 20, 241-250.

Kensinger, E. A., \& Schacter, D. L. (2006). Amygdala activity is associated with the successful encoding of item, but not source, information for positive and negative stimuli. Journal of Neuroscience, 26, 2564-2570.

MATHER, M. (2007). Emotional arousal and memory binding: An objectbased framework. Perspectives on Psychological Science, 2, 33-52.

Mather, M., Mitchell, K. J., Raye, C. L., NovaK, D. L., Greene, E. J., \& Johnson, M. K. (2006). Emotional arousal can impair feature binding in working memory. Journal of Cognitive Neuroscience, $\mathbf{1 8}$, 614-625.

Mather, M., \& NeSmith, K. (2008). Arousal-enhanced location memory for pictures. Journal of Memory \& Language, 58, 449-464.

Mealey, L., Daood, C., \& Krage, M. (1996). Enhanced memory for faces of cheaters. Ethology \& Sociobiology, 17, 119-128.

Mehl, B., \& Buchner, A. (2008). No enhanced memory for faces of cheaters. Evolution \& Human Behavior, 29, 35-41.

Murnane, K., \& Bayen, U. J. (1998). Measuring memory for source: Some theoretical assumptions and technical limitations. Memory \& Cognition, 26, 674-677.

NaIRNE, J. S., \& PandeIRada, J. N. S. (2008a). Adaptive memory: Is survival processing special? Journal of Memory \& Language, 59, 377385.

Nairne, J. S., \& Pandeirada, J. N. S. (2008b). Adaptive memory: Remembering with a stone-age brain. Current Directions in Psychological Science, 17, 239-243.

Nairne, J. S., Pandeirada, J. N. S., \& Thompson, S. R. (2008). Adaptive memory: The comparative value of survival processing. Psychological Science, 19, 176-180.

Nairne, J. S., Thompson, S. R., \& Pandeirada, J. N. S. (2007). Adaptive memory: Survival processing enhances retention. Journal of Experimental Psychology: Learning, Memory, \& Cognition, 33, 263273.

OCHSNER, K. N. (2000). Are affective events richly recollected or simply familiar? The experience and process of recognizing feelings past. Journal of Experimental Psychology: General, 129, 242-261.

ODA, R. (1997). Biased face recognition in the prisoner's dilemma game. Evolution \& Human Behavior, 18, 309-315.

Öhman, A., Lundqvist, D., \& Esteves, F. (2001). The face in the crowd revisited: A threat advantage with schematic stimuli. Journal of Personality \& Social Psychology, 80, 381-396.

Peeters, G., \& Czapinski, J. (1990). Positive-negative asymmetry in evaluations: The distinction between affective and informational negativity effects. In W. Stroebe \& M. Hewstone (Eds.), European review of social psychology (Vol. 1, pp. 33-60). Chichester, U.K.: Wiley.

Pratto, F., \& John, O. P. (1991). Automatic vigilance: The attentiongrabbing power of negative social information. Journal of Personality \& Social Psychology, 61, 380-391.

Rossmann, P. (1984). On the forgetting of word associations: Parkin et al. reconsidered. Psychological Research, 45, 377-388.

Rothbart, M., \& PARK, B. (1986). On the confirmability and disconfirmability of trait concepts. Journal of Personality \& Social Psychology, 50, 131-142.

ROTHKEGEL, R. (1999). AppleTree: A multinomial processing tree modeling program for Macintosh computers. Behavior Research Methods, Instruments, \& Computers, 31, 696-700.

Rozin, P., \& Fallon, A. E. (1987). A perspective on disgust. Psychological Review, 94, 23-41.

Schienle, A., Walter, B., Stark, R., \& Vaitl, D. (2002). Ein Fragebogen zur Erfassung der Ekelempfindlichkeit (FEE) [A questionnaire for the assessment of disgust sensitivity]. Zeitschrift für Klinische Psychologie und Psychotherapie: Forschung und Praxis, 31, 110-120.

SCHMIDT, S. R. (1991). Can we have a distinctive theory of memory? Memory \& Cognition, 19, 523-542.

SKowRONSKI, J. J., \& CARLSTON, D. E. (1989). Negativity and extremity biases in impression formation: A review of explanations. Psychological Bulletin, 105, 131-142.

Smith, A. P. R., Dolan, R. J., \& RugG, M. D. (2004). Event-related potential correlates of the retrieval of emotional and nonemotional context. Journal of Cognitive Neuroscience, 16, 760-775.

Snodgrass, J. G., \& Corwin, J. (1988). Pragmatics of measuring rec- 
ognition memory: Applications to dementia and amnesia. Journal of Experimental Psychology: General, 117, 34-50.

SPANIOL, J., \& BAYEN, U. J. (2002). When is schematic knowledge used in source monitoring? Journal of Experimental Psychology: Learning, Memory, \& Cognition, 28, 631-651.

Stahl, C., \& KLauer, K. C. (2007). HMMTree: A computer program for latent-class hierarchical multinomial processing tree models. Behavior Research Methods, 39, 267-273.

ToRonchuK, J. A., \& Ellis, G. F. R. (2007). Disgust: Sensory affect or primary emotional system? Cognition \& Emotion, 21, 1799-1818.
Trivers, R. L. (1971). The evolution of reciprocal altruism. Quarterly Review of Biology, 46, 35-57.

WASON, P. C. (1968). Reasoning about a rule. Quarterly Journal of Experimental Psychology, 20, 273-281.

Wentura, D., Rothermund, K., \& BaK, P. (2000). Automatic vigilance: The attention-grabbing power of approach- and avoidancerelated social information. Journal of Personality \& Social Psychology, 78, 1024-1037.

ZaJONC, R. B. (1968). Attitudinal effects of mere exposure. Journal of Personality \& Social Psychology, 9, 1-27.

APPENDIX

Parameter Estimates for Old-New Discrimination, Source Memory, and Guessing Biases

\begin{tabular}{|c|c|c|}
\hline \multirow[b]{2}{*}{ Parameter } & \multicolumn{2}{|c|}{ Parameter Estimate } \\
\hline & Experiment 1 & Experiment 2 \\
\hline \multicolumn{3}{|c|}{ Old-New Discrimination } \\
\hline$D_{-}=D_{\sim}=D_{+}=D_{\mathrm{N}}$ & .58 & .54 \\
\hline \multicolumn{3}{|c|}{ Source Memory } \\
\hline$d_{-}$ & .37 & .31 \\
\hline$d_{\sim}$ & .00 & .01 \\
\hline$d_{+}$ & .17 & .12 \\
\hline \multicolumn{3}{|c|}{ Guessing Biases } \\
\hline$a_{ \pm}$ & .60 & .63 \\
\hline$a_{-}$ & .47 & .46 \\
\hline$b$ & .29 & .30 \\
\hline$g_{ \pm}$ & .51 & .54 \\
\hline$g_{-}$ & .55 & .54 \\
\hline \multicolumn{3}{|c|}{$\begin{array}{l}\text { Note }-D \text {., probability of correctly identifying a face as old } \\
\text { (for previously presented faces) or new (for new faces); } d_{-} \text {, } \\
d_{\sim}, d_{+} \text {, conditional probability of correct source identifica- } \\
\text { tion given correct old-new discriminations for faces encoun- } \\
\text { tered in negative, neutral, and positive contexts, respectively; } \\
a_{ \pm} \text {, probability of guessing that a recognized face was en- } \\
\text { countered in a valenced context; } a_{-} \text {, probability of guessing } \\
\text { that a recognized face was encountered in a negative context; } \\
b, \text { probability of guessing that a nonrecognized face was old; } \\
g_{ \pm} \text {, probability of guessing that a nonrecognized face that } \\
\text { was guessed to be old was encountered in a valenced con- } \\
\text { text; } g_{-} \text {, probability of guessing that a nonrecognized face } \\
\text { that was guessed to be old was encountered in a negative } \\
\text { context. }\end{array}$} \\
\hline
\end{tabular}

(Manuscript received February 3, 2009;

revision accepted for publication April 13, 2009.) 